\title{
Metal-to-insulator transition and threshold electric field for SDW depinning in (TMTSF) 2 X under pressure
}

\author{
M. NAGASAWA, T. SAMBONGI, K. NOMURA and H. ANZAI* \\ Department of Physics, Hokkaido University, Sapporo 060, Japan \\ * Department of Materials Sciences, Himeji Institute of Technology, Kanagi, Kamigohri Hyogo 678-12, \\ Japan
}

\begin{abstract}
Metal-to-insulator transition temperature $\left(T_{M Y}\right)$ and the activation energy $\left(\mathrm{E}_{\mathrm{A}}\right)$ in the SDW phase of (TMTSF) ${ }_{2} \mathrm{ASF}_{6}$ and (TMTSF) ${ }_{2} \mathrm{PF}_{6}$ were determined under pressure. Pressure dependence of $2 \mathrm{E}_{\mathrm{A}} / \mathrm{k}_{\mathrm{B}} \mathrm{T}_{\mathrm{MI}}$ near the critical pressure of the SDW phase, is compared with the mean-field theory by Yamaji. It was found that $T_{M I}$ is not equal to the normal-to-SDW transition temperature but lower than $\mathrm{T}_{\text {SDw }}$. Non-linear conduction was observed in a narrow temperature range above $\mathrm{T}_{\mathrm{MI}}$.

\section{Introduction}

In (TMTSF) ${ }_{2} \mathrm{ASF}_{6}$ and (TMTSF) ${ }_{2} \mathrm{PF}_{6}$ the spin density wave (SDW) state has been observed below $\mathrm{T}_{\text {SDw }} \sim 10^{1} \mathrm{~K}$ at the ambinet pressure. $\mathrm{T}_{\text {SDw }}$ is lowered under pressure. Brusetti et al. [1] showed from conductivity measurement that, in $\left(\right.$ TMTSF) ${ }_{2} A_{S F_{6}}, T_{S D r}=12 \mathrm{~K}$ at the ambient pressure and that the critical pressure $\mathrm{P}_{c}$ of the SDW phase is $\sim 10.5 \mathrm{~kb}$. Takahashi et al. [2] performed ${ }^{2} \mathrm{H}-\mathrm{NMR}$ experiment in (TMTSF) ${ }_{2} \mathrm{PF}_{6}$ under pressure and found that the SDW gap parameter at $\mathrm{T}=0, \Delta_{0}(\mathrm{~T}=0)$, is insensitive to pressure while $\mathrm{T}_{\mathrm{sDw}}$ is lowered with pressure. Their results are in good agreement with the the mean-field theory first developed by Yamaji [3].

In the simplified model of electrons in (TMTSF) ${ }_{2} X$, a finite transverse transfer energy $t_{b}$ is added to the one-dimensional dispersion. The Fermi surface is open but warped. In such a system, the ground state is the SDW and the energy gap developes over the entire area of the Fermi surface as long as $t_{b}$ is smaller than the critical value. In the SDW state the band edges above and below the Fermi level are not constant but are modelated with amplitude $\varepsilon_{0}$, where $\varepsilon_{0} \propto t_{b}{ }^{2}$ is the measure of imperfect nesting of the Fermi surface. The sDW state is stabilized as long as $\varepsilon_{0}$ is smaller than the gap parameter at $0 \mathrm{~K}, \Delta_{0}(T=0)$. While $\Delta_{0}(T=0)$ itself is insensitive to $\varepsilon_{0}$, the SDW transition temperature $T_{S D W}$ decreases with increasing $\varepsilon_{0}$. The thermal activation evergy $E_{A}$ is equal to $\Delta_{0}(0)-\varepsilon_{0}$ which is the minimum energy between occupied and unoccupied bands; it decreases with increasing $\varepsilon_{0}$. Therefore, $E_{A}$ is a useful parameter of the degree of imperfectness of the Fermi surface or the two-dimensionality of the systen. Yamaji predicted that the semimetallic SDW state appears above $\mathrm{T}_{M I}<\mathrm{T}_{S D}$ and that the temperature difference $T_{S D W}-T_{M I}$ increases with increasing $\varepsilon_{0}$. From numerical calculation, Yamaji obtained that, as $\varepsilon_{0} \rightarrow \Delta_{O}\left(E_{A} \rightarrow 0\right), 2 E_{A} / k_{B} T_{S D G} \rightarrow 0$ and $2 \mathrm{E}_{\mathrm{A}} / \mathrm{k}_{\mathrm{B}} \mathrm{T}_{\mathrm{MI}} \rightarrow 1.4$, respectively.

Because it is expected that $\varepsilon_{0}$ increases with increasing the applied pressure, theoretical predictions have been compared with experimental results under pressure. We determined $T_{M I}$ and $E_{A}$ from ohmic conductivity under pressure. The
\end{abstract}


experimental results, especially the ratio $2 \mathrm{E}_{\mathrm{A}} / \mathrm{k}_{\mathrm{B}} \mathrm{T}_{\mathrm{M}}$ around $\mathrm{P}_{\mathrm{C}}$, are compared with the mean-field theory to determine whether $T_{M I}$ is equal to or different from $\mathrm{T}_{\text {SDw. }}$. In addition, observation of non-linear conductivity in the temperature range near $\mathrm{T}_{c}$ is reported and discussed.

\section{Experiment}

Single crystals of (TMTSF) ${ }_{2} \mathrm{AsF}_{6}$ and (TMTSF) ${ }_{2} \mathrm{PF}_{6}$ were grown by the standard electrochemical method. Annealed gold wires of $10 \mu \mathrm{m}$ diameter were used as electrical leads. They were attached with silver paste to Au pads evaporated on the sample. The current contacts covered the whole areas of both ends of the crystal for uniform current density. Mixture of kerosene and transformer oil with a ratio 1:1 was used as pressure medium in a clamp type bomb. In this experiment we have not observed resistance jump which frequently occurs during cooling at the ambient pressure.

We determined the T-P diagram below $4.2 \mathrm{~kb}$ from the superconducting transition temperature of indium manometer inside the bomb. Above $5 \mathrm{~kb}$, the actual pressure was estimated from the phase diagram of (TMTSF) ${ }_{2} A_{S F}$ determined by Brusetti et al. [1]. The metal-insulator transition temperature $\mathrm{T}_{M I}$ was determined from the peak of $\left|\mathrm{d}\left(\ln \sigma_{0}\right) / \mathrm{d}(1 / \mathrm{T})\right|$ vs $\mathrm{T}$ curve, where $\sigma_{0}$ is the the ohmic conductance. The activation energy $\left(E_{A}\right)$ was determined from the Arrhenius plot of $\sigma_{\circ}$ below $2 \mathrm{~K}$, i.e., below $\mathrm{T}_{C} / 2$ and $2 \mathrm{E}_{\mathrm{A}} / 3$ at the highest pressure in this work. Because of the non-linear increase of the conductivity in the SDW state, we measured the pulse conductivity and compared with the dc conductivity to determine $\sigma_{\circ}$.

\section{Result and discussion}

The ohmic conductance $\sigma_{o}$ was measured carefully by repeated cooling and heating cycles, to find limit of temperature difference between the sample and the thermometer attached to the bomb. The values of $T_{M I}$ determined from the heating and cooling runs were in agreement with each other within $\pm 0.2 \mathrm{~K}$. The limit of uncertainty of $\mathrm{E}_{\mathrm{A}}$ is smaller than $\pm 5 \%$. The applied pressure was increased from $1 \mathrm{~kb}$ up to $9 \mathrm{~kb}$ and then decreased down to $1.5 \mathrm{~kb}$. There was no hysteresis with changing pressure in the $E_{A}-T_{M x}$ curve and the peak height of $\left|\mathrm{d}\left(\ln \sigma_{\mathrm{o}}\right) / \mathrm{d}(1 / \mathrm{T})\right|-\mathrm{T}$ curve was reproducible. As shown in Fig.1 that peak is progressively broadened with pressure but does not vanish even at the highest pressure of $P \sim 9 \mathrm{~kb}$, in contrast with the results by Brusetti et al.[1] who could not observe the peak above $8 \mathrm{~kb}$.

In both (TMTSF) ${ }_{2} \mathrm{ASF}_{6}$ and (TMTSF) ${ }_{2} \mathrm{PF}_{6}$, both $\mathrm{T}_{M I}$ and $E_{A}$ decrease with increasing pressure. From Fig.2, the pressure coefficients of $\mathrm{T}_{M I}$ and $\mathrm{E}_{\mathrm{A}}$ of (TMTSF) ${ }_{2} \mathrm{AsF}_{6}$ are $\mathrm{dT}_{\mathrm{MI}} / \mathrm{dP}=-0.83 \mathrm{~K} / \mathrm{kb}$ and $\mathrm{dE}_{\mathrm{A}} / \mathrm{dP}=-2.1 \mathrm{~K} / \mathrm{kb}$ below $4 \mathrm{~kb}$, respectively. We have not determined $\mathrm{T}_{M I}$ of (TMTSF) ${ }_{2} \mathrm{AsF}_{6}$ at the ambient pressure directly. From extrapolation to $P=0, T_{M I}(P=0)=13.0 \mathrm{~K}$. These values should be compared with $\mathrm{dT}_{\mathrm{M} X} / \mathrm{dP} \sim-0.6 \mathrm{~K} / \mathrm{kb}$ and $\mathrm{T}_{\mathrm{MI}}(\mathrm{P}=0) \sim 12 \mathrm{~K}$ obtained by Brusetti et al. [1] in $\left(\right.$ TMTSF) ${ }_{2} \mathrm{AsF}_{6}$.

In Fig. 3 the ratio $2 \mathrm{E}_{\mathrm{A}} / \mathrm{k}_{\mathrm{B}} \mathrm{T}_{\mathrm{MI}}$ is plotted, not against pressure, but against $\mathrm{E}_{\mathrm{A}}$ because it is more convenient to compare the present results with the mean-field theory. From extrapolation to $\mathrm{P}=0,2 \mathrm{E}_{\mathrm{A}} / \mathrm{k}_{\mathrm{B}} \mathrm{T}_{\mathrm{MI}}$ in both (TMTSF) ${ }_{2} \mathrm{AsF}_{6}$ and (TMTSF) ${ }_{2} \mathrm{PF}_{6}$ at the ambient pressure is $\sim 3.0$, which is much smaller than 3.53 expected from the mean-field theory when $\varepsilon_{0}=0$. With decreasing $E_{A}, 2 E_{A} / k_{B} T_{M I}$ decreases and its limiting value at $E_{A}=0$ is approximately 1.3 . Even if we take the limits of uncertainty of both $T_{M I}$ and $E_{A}$ into consideration, it cannot be expected that $2 \mathrm{E}_{\mathrm{A}} / \mathrm{k}_{\mathrm{B}} \mathrm{T}_{M I}$ approaches zero. Though the definition of $\mathrm{T}_{M I}$ used in this paper is rather arbitrary, the above conclusion does not change if $T_{M I}$ is defined in a different way. For example, The temperature difference between the peak in $\left|\mathrm{d}\left(\ln \sigma_{0}\right) / \mathrm{d}(\mathrm{T})\right|$ and that in $\left|\mathrm{d}\left(\ln \sigma_{0}\right) / \mathrm{d}(1 / \mathrm{T})\right|$ does not exceed $0.2 \mathrm{~K}$. We have not extended measurements on (TMTSF) ${ }_{2} \mathrm{PF}_{6}$ to higher pressure but it is expected that the ratio remains finite at high pressure as in (TMTSF) ${ }_{2} A_{s} F_{6}$.

According to the mean-field theory, maxima of the density of states in the SDW 


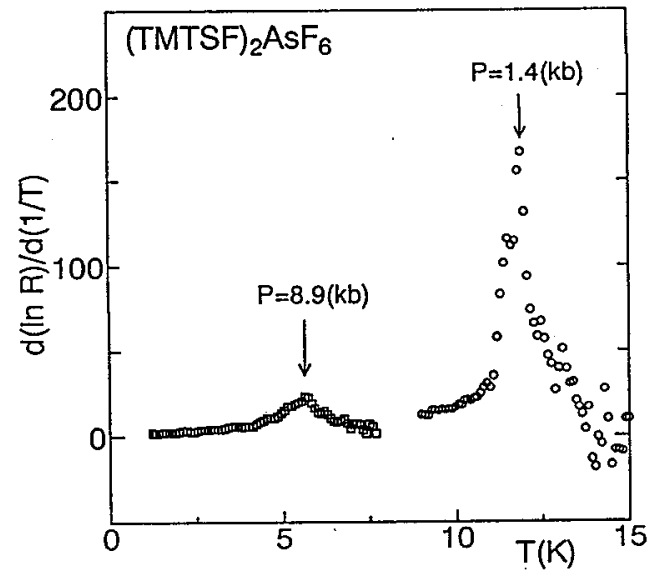

Fig.1 $\left|\mathrm{d}\left(\ln \sigma_{\mathrm{o}}\right) / \mathrm{d}(1 / \mathrm{T})\right|$ vs. temperature. ( $\sigma_{o}:$ ohmic conductivity)

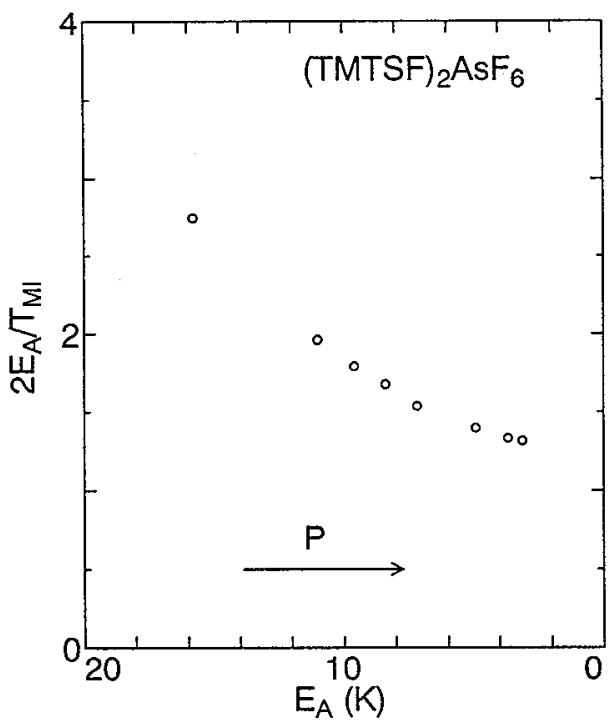

Fig. 3. Ratio $2 \mathrm{E}_{\mathrm{A}} / \mathrm{k}_{\mathrm{B}} \mathrm{T}_{M I}$ vs. $\mathrm{E}_{\mathrm{A}}$.

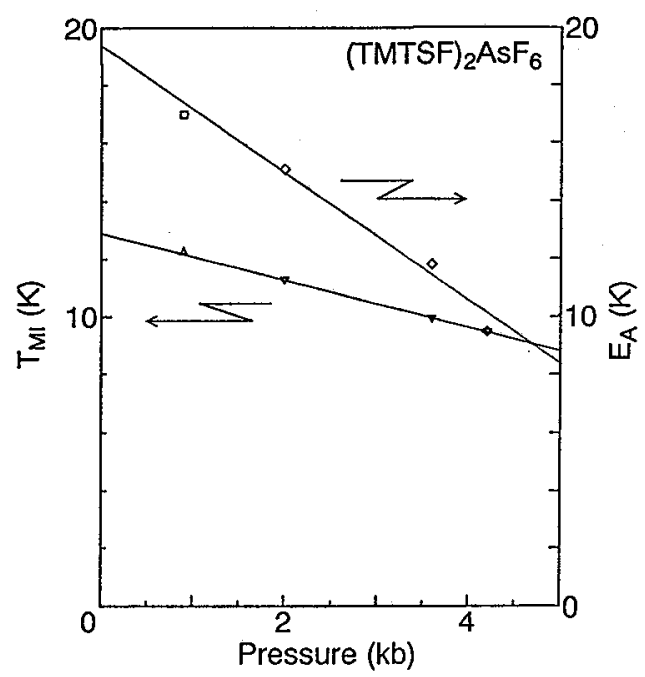

Fig.2 Metal-insulator transtition temperature $T_{M I}$ and activation energy $\mathrm{E}_{\mathrm{A}}$ under pressure. 
state are located at $E= \pm\left(\Delta_{0}+\varepsilon_{0}\right)$. Their magnitudes decrease with increasing $\varepsilon_{0}$. on the other hand, the density of states at $E= \pm\left(\Delta_{0}-\varepsilon_{0}\right)$ is finite irrespective of $\varepsilon_{0}$. Thus almost all the carriers are excited between the top of occupied band and bottom of unoccupied band; the activation energy $E_{A}$ obtained below $2 \mathrm{~K}$ is approximately equal to $\Delta_{\mathrm{o}}-\varepsilon_{\mathrm{o}}$.

If there are impurity levels between occupied and unoccupied bands, $E_{A}$ cannot be determined from the Arrhenius plot, or it would be smaller than $\Delta_{o}-\varepsilon_{o}$ even if the Arrhenius plot is approximated by a linear curve. In fact, we observed nonlinear curves in some (TMTSF) ${ }_{2} \mathrm{PF}_{6}$ samples. In such cases, we obtained $\mathrm{E}_{\mathrm{A}}$ from $\sigma_{0}-\sigma_{i}$ instead of $\sigma_{o}$, where $\sigma_{i}$ is a constant independent of temperature. It was assumed that the carriers provided by impurities contribute to $\sigma_{1}$. These $\mathrm{E}_{\mathrm{A}}$ values are in agreement with those determined from other samples which show the regular linear Arrhenius curves.

We found that the liniting value of $2 \mathrm{E}_{A} / \mathrm{k}_{B} \mathrm{~T}_{M I}$ is 1.3 , which is close to 1.4 calculated by Yamaji [3]. Therefore, $T_{M I}$ determined experimentally is not equal to $\mathrm{T}_{\text {SDW }}$ but lower than $\mathrm{T}_{\text {SDw }}$.

In several samples of both (TMTSF) ${ }_{2} \mathrm{AsF}_{6}$ and (TMTSF) $2 \mathrm{PF}_{6}$, non-linear conduction was observed above $\mathrm{T}_{\mathrm{Mr}}$. In some of them a finite threshold field was found and it decreases with increasing temperature and vanishes at a temperature slightly higher than $\mathrm{T}_{\mathrm{MI}}$. We expect that $\mathrm{T}_{\mathrm{SD}}$ can be determined by extrapolation of the threshold field to zero.

\section{References}

[1] Brusetti,R., Ribault,M., Jerome,D. and Bechgaard,K., J.Physique, 43, (1982) 801

[2] Takahasi,T.,Kawamura,H., Ohyama,T., Maniwa,Y., Murata,K. and Saito,G., J.Phys.Soc.Japan 58, (1989) 703

[3] Yamaji,K., J.Phys.Soc.Japan 51, (1982) 2787 